\title{
DESIGN AND DEVELOPMENT OF A TUNABLE PROPORTIONAL INTEGRAL DERIVATIVE (TPID) CONTROLLER FOR A ROBOTIC ARM
}

Akaninyene M. Joshua Ph.D and Prof. Eneh I.I.

Dept of Electrical and Electronics Engineering, Enugu State of Science and Technology, Enugu State.

\begin{abstract}
The main targets in designing control systems are stability, good disturbance rejection, and small tracking error. Several industrial robot manipulators are controlled by linear methodologies such as Proportional-Derivative (PD) controller, Proportional-Integral (PI) controller or Proportional-Integral-Derivative (PID) controllers. In this work, a Tunable Proportional Integral Derivative (TPID) controller is proposed for the control of a robotic arm. This tuning method is an attempt to obviate the shortcomings of the conventional PID controller where the proportional gain KP, integral gain $\mathrm{KI}$ and derivative gain $\mathrm{KD}$, are fixed. The proposed controller provides opportunity for tuning the PID to be able to control the nonlinear movements and operations of a robot. The robot arm manipulator can be tuned as desired to provide control measures to enhance stability and suppress vibrations arising from robot arm operation. Simulation results showed an improvement over conventional PID controller for robotic arm manipulator.
\end{abstract}

Keywords: Proportional-Derivative (PD) controller, Proportional-Integral (PI) controller, Proportional-Integral-Derivative (PID), Tunable Proportional Integral Derivative (TPID) Controller.

\subsection{Introduction}

A controller is a device which can sense information from linear or nonlinear system (e.g., the proposed robot manipulator) to improve the systems performance (Cheng et al, 2008). The main targets in designing control systems are stability, good disturbance rejection, and small tracking error (D'Azzo et al, 2003). Several industrial robot manipulators are controlled by linear methodologies such as Proportional-Derivative (PD) controller, ProportionalIntegral (PI) controller or Proportional-Integral-Derivative (PID) controllers (Farzin et al, 2012). According to Fallahi et al, (2011), the proportional-integral-derivative (PID) controller is widely used in many control applications because of its simplicity and effectiveness. The use of PID control has been a long history in the field of control engineering. The three controller gain parameters namely, proportional gain KP, integral gain KI, and derivative gain KD, are usually fixed. The disadvantage of the fixed PID controller is poor capability in dealing with system uncertainty, that is, parameter variations and external disturbance.

To solve this problem the PID tuning method was introduced (Skogestad, 2001) justified the use of PID controller by saying that hundreds, if not thousands, of literatures have been written on tuning of PID controllers, and one must question the need for another one. Inyiama and Emmanuel, (2015), seconded the opinion with justification that PID controller is by far the most widely used control algorithm in the process industry, and that improvements in tuning of PID controllers will have a significant practical impact but this have to be justified in this work in the results. 
Adaptive controller can be seen as any control framework that can monitor its operation and make alterations on its dynamics and processes as to enhance system performance (Ehsani, 2007). Fundamentally, there are four essential adaptive controllers according to Ehsani, (2007), viz; (1) Dual Control, (2) Gain Scheduling, (3) Model Reference Adaptive Control (MRAC), and (4) Self-Tuning Regulators (STR).

Gain Scheduling: This approach is applied to nonlinear control systems. According to Mathworks, (2017), it deploys a group of linear controllers and individually provide adequate control for various different operating points of the control system (Jarzebowska, 2012). This implies that small set of operating points are considered when designing a Gain Scheduling control system. In other words, this approach is deployed when supplementary variables that respond well to the characteristics of the process dynamics in some systems can be measured, which can be utilized to change or update the parameters of the controller system. This process is very vital because it can be used to allow for changes in process gain (Ehsani, 2007; Astrom and Wittenmark, 1989).

MRAC: Moussavi, Alasvandi, Javadi and Morad, (2014), described MRAC as an adaptive servo architecture in which the desired output or performance is defined in terms of a reference model, which provides desired response to the reference signal (Ezzeldin, van den Bosch and Waarsing, 2009). Generally speaking, MRAC is a unit of four parts namely; the plant to be controlled, containing unknown parameters, a reference model for constantly specifying the desired output of the control system in a compact way, a feedback control law made up of parameters that can be adjusted and the NN controller. Parameter information is extracted by the adaptation law of MRAC systems from the tracking errors (Ehsani, 2007).

\subsection{Review of Related Works}

Reviews of some related works provided more insight to undertake the present work for better and more reliable results. These are outlined as follows.

Arulmozhiand Baskar(2017) presented a work on Development of Various Tunable PID Controllers for A Robotic Arm System.Based on error criteria and time domain specifications for controlling the angular position of a robotic arm, analyses and comparison of different types of controllers such as P, PI and PID was carried out.Using different tuning techniques like Ziegler- Nichols and TyreusLuyben, the proportional gain (Kp), integral gain (Ki) and derivative gain $(\mathrm{Kd})$ for the controller were determined. Time domain specifications and error criteria of various controllers were measured and compared in these techniques, taking the PID structure into consideration for analysis.Bytuning all the controller parameter with and without disturbances in MATLAB environment,the proposed techniques were simulated. Results show the effectiveness of the proposed scheme.The time domain specifications proved that $\mathrm{ZN}$ tuned PID controller produced minimum settling time, showing the performance of IAE and ISE under the error criterion better for the proposed controller.

Zakia et al, (2019), in PID-SMC Controller for A 2-DOF Planar Robot, considered a Sliding Mode Controlleras a robust control method. A combination of SMC and traditionalproportional-integral-differential (PID) control scheme for a 2-degree of freedom (2-DOF) planar manipulator was considered. Using the PID component parameters of the manipulator, a sliding mode surface for the SMC control was designedsince the PID control does not require precise system modeling. While the SMC control ensures fast convergence towards the sliding mode surface, the PID controller adjusts the errors of the closed loop system. This made the hybrid controller possess features of both control schemes and obtained better system stability. 
Joyo et al, (2019) in Optimized Proportional-Integral-Derivative Controller for Upper Limb Rehabilitation Robot,proposed a nature inspired meta-heuristic optimization technique to tune aproportional-integral-derivative (PID) controller for a robotic arm exoskeleton RAX-1. Undesirable large overshoots forrehabilitation applications are produced usingZieglerNichols conventional tuning of PID controllers. To avoid this, nature inspired algorithms was proposed to improve PID controllers' performance.A 2-DOF PID control system was optimized offline using particle swarm optimization (PSO) and artificial bee colony (ABC). Several simulations were carried out to validate the effectiveness of the proposed ABC-PID method in comparison with the PSO-PIDand a classical PID controller tuned using the Zeigler-Nichols method.Various investigations were carried out such as determination of system performance with respect to maximum overshoot, rise and settling time as well as using maximum sensitivity function under disturbance. Investigation results showed thatthe $\mathrm{ABC}-\mathrm{PID}$ was more robust and outperformed other tuning techniques, demonstrating the effective response of the proposed technique for a robotic manipulator.Also, the ABC-PID controller was implemented on the hardware setup of RAX-1 and the response during exercise showed minute overshoot with lower rise and settling times compared to PSO and Zeigler-Nichols-based controllers.

These reviews showed improvements of the various techniques over conventional methods of tuning the controllers thus supporting the need for this work.

\subsection{Methodology}

\subsection{Controller Design}

Controller is a device which can sense information from linear or nonlinear system (e.g., the proposed robot manipulator) to improve the systems performance (Cheng et al, 2008). The main targets in designing control systems are stability, good disturbance rejection, and small tracking error (D'Azzo et al, 2003). Several industrial robot manipulators are controlled by linear methodologies e.g., Proportional-Derivative (PD) controller, Proportional- Integral (PI) controller or Proportional- Integral-Derivative (PID) controllers (Farzin et al, 2012). According to Fallahi et al, (2011), the proportional-integral-derivative (PID) controller is widely used in many control applications because of its simplicity and effectiveness. The use of PID control has been a long history in the field of control engineering, the three controller gain parameters, proportional gain $\mathrm{KP}$, integral gain $\mathrm{KI}$, and derivative gain $\mathrm{KD}$, are usually fixed. The disadvantage of the fixed PID controller is poor capability of dealing with system uncertainty, i.e., parameter variations and external disturbance.

To solve this problem the PID tuning method was introduced (Skogestad, 2001) justified the use of PID controller by saying that hundreds, if not thousands, of literatures have been written on tuning of PID controllers, and one must question the need for another one. Inyiama and Emmanuel, (2015), seconded the opinion with justification that PID controller is by far the most widely used control algorithm in the process industry, and that improvements in tuning of PID controllers will have a significant practical impact but this have to be justified in this work in the results.

Various Proportional-Integral-Derivative (PID) controller tuning methods such as the manual, Ziegler Nichols Youns et al (2013), software tool (Inyama, 2015), fuzzy logic (Kumar and Raja, 2014), Genetic Algorithm (GA) (Youns et al, 2013;Muhammad, 2013) methods have been proposed. However, none of these controllers are without its advantages and limitations. It is difficult to achieve optimal performance using manual and Zeigler Nichols methods. Fuzzy logiccan be used to achieve desired optimal performance but they lack the parameters to evaluate the disturbance rejection settling time which helps to determine how fast the system can cancel or reject disturbance in harsh environments. On the other hand, the software tool method for PID can be used to achieve the desired performance and good 
disturbance rejection settling time but most times the PID controller can become aggressive and saturate the DC motor. Hence there is need for a controller that will complement the PID, canceling this non linearity and control the manipulator joint response precisely at different time series. This will be actualized and implemented using the artificial neural network. Therefore this work will deploy a hybrid of tuner Proportional Integral Derivative and Artificial Neural Network (TPID and NN) controllers to achieve precision in motion control thereby reducing vibration to the bearable level.

\subsection{Design of the tunable P-I-D controller}

To develop a tunable PID controller, the Proportional (P) Integral (I) and Derivative (D) controllers must be designed and combined together before it can be tuned. The proportional controller design is given as the equation (Inyiama, 2017);

$\mathrm{P}_{\text {OUT }}=\mathrm{K}_{\mathrm{P}} \mathrm{e}(\mathrm{t})+\mathrm{P}_{0}$

Where $\mathrm{P}_{\text {out }}$ : controller output; $\mathrm{K}_{\mathrm{p}}$ : Proportional gain;e(t): Instantaneous error at $\mathrm{T}(\mathrm{time})$.

But $\mathrm{e}(\mathrm{t})=\mathrm{SP}-\mathrm{PV}$

Where SP: Set point; PV: Process variable; $\mathrm{P}_{0}$ :offset value

The equation of the integral controller is presented as (Pranaya, 2017);

$\mathrm{P}(\mathrm{t})=\mathrm{K}_{\mathrm{i}} \int E_{P}(t) d t+\mathrm{P}_{0}$

Where $\mathrm{P}(\mathrm{t})$ : output of the integral controller; Ki: Integral gain; $\mathrm{E}_{\mathrm{p}}(\mathrm{t})$ : error of the system; $\mathrm{P}_{0}$ : offset value.

Derivative function is given by (Inyama, 2015);

$\mathrm{P}(\mathrm{t})=\mathrm{K}_{\mathrm{d}} \frac{d E_{P}}{d t}+\mathrm{P}_{0}$

Where $\mathrm{P}(\mathrm{t})$ : derivative controller output; $\mathrm{K}_{\mathrm{d}}$ : derivative gain; $\mathrm{P}_{0}$ : offset value; $\mathrm{E}_{\mathrm{p}}$ : error of the system.

Eneh et al. (2019) and Ngoc et al., (2009), designed the PID controller combining the three mathematical functions modeled by equation $3.57,3.59$ and 3.60 to produce the controller as in the following equation;

$\mathrm{u}=\mathrm{K}_{\mathrm{p}}(\mathrm{br}-\mathrm{y})+\mathrm{K}_{\mathrm{i}} \mathrm{IF}(\mathrm{z})(\mathrm{r}-\mathrm{y})+{ }_{\mathrm{K}} \mathrm{d}_{\mathrm{T}} \mathrm{f}_{+\mathrm{DF}(\mathrm{z})}(\mathrm{cr}-\mathrm{y})$.

where;

$K p=$ proportional gain

$K i=$ integrator gain

$K_{d}=$ derivative gain

$T_{f}=$ derivative filter time

$b=$ set point weight on proportional term

$c=$ set point weight on derivative term

\section{Tuning the PID Controller}

To tune and improve the PID controller, we set the gain of the undesired control output to zero. This is achieved using the following tuning parameters;

Proportional gain, $\boldsymbol{K}_{p}$ :Larger values typically mean faster response since the larger the error, the larger the Proportional term compensation. An excessively large proportional gain will lead to process instability and oscillation. 
Integral gain, $\boldsymbol{K}_{\boldsymbol{i}}$ :Larger values imply steady state errors are eliminated more quickly. The trade-off is larger overshoot: any negative error integrated during transient response must be integrated away by positive error before steady state is reached.

Derivative gain, $\boldsymbol{K}_{\boldsymbol{d}}$ : Larger values decrease overshoot, but slows down transient response and may lead to instability due to signal noise amplification in the differentiation of the error. The proportional term is given as

$$
\mathrm{P}_{\text {out }}=\mathrm{K}_{\mathrm{p}} \mathrm{e}(\mathrm{t})
$$

Where $P_{\text {out }}$ : Proportional term of output; $K_{p}$ : Proportional gain, a tuning parameter; $e:$ Error $=S P-P V ; t:$ Time or instantaneous time (the present)

The Integral term is given as:

$$
I_{\text {out }}=K_{i} \int_{0}^{t} e(\tau) d \tau
$$

Where is the $I_{\text {out }}$ Integral term of output; $K_{i}$ : Integral gain, which is a tuning parameter; $e:$ Error $=S P-P V$, where $t$ is the time or instantaneous time (the present); $\tau$ : A dummy integration variable The derivative term is given by:

$$
\mathrm{D}_{\text {out }}=\mathrm{K}_{\mathrm{d}} \frac{d e(t)}{d t}
$$

$D_{\text {out }}$ is the Derivative term of output; $K_{d}$ : Derivative gain, is also a tuning parameter; $e$ is a given in equation (3.64) and $t$ as well is the time or instantaneous time (the present) The Proportional, Integral, and Derivative terms are summed to calculate the output of the tuned PID controller $u(t)$ as follows;

$$
u(t)=\mathrm{MV}(\mathrm{t})=K_{p} e(t)+K_{i} \int_{0}^{t} e(\tau) d \tau+K_{d} \frac{d e(t)}{d t}
$$

The developed mathematical models of the PID and robot arm are in time domain and as a result, cannot be simulated using Simulink tool. There is need to develop the models in the frequency domain. To achieve this, the mathematical model of the PID derived in equation (3.9), which is the transfer function and that of the robot arm was used to develop the Simulink models. The PID was integrated with the robot arm to carry out real-time simulation.

The developed robot arm and PID Simulink models are as shown in Figures 3.1 and 3.2.

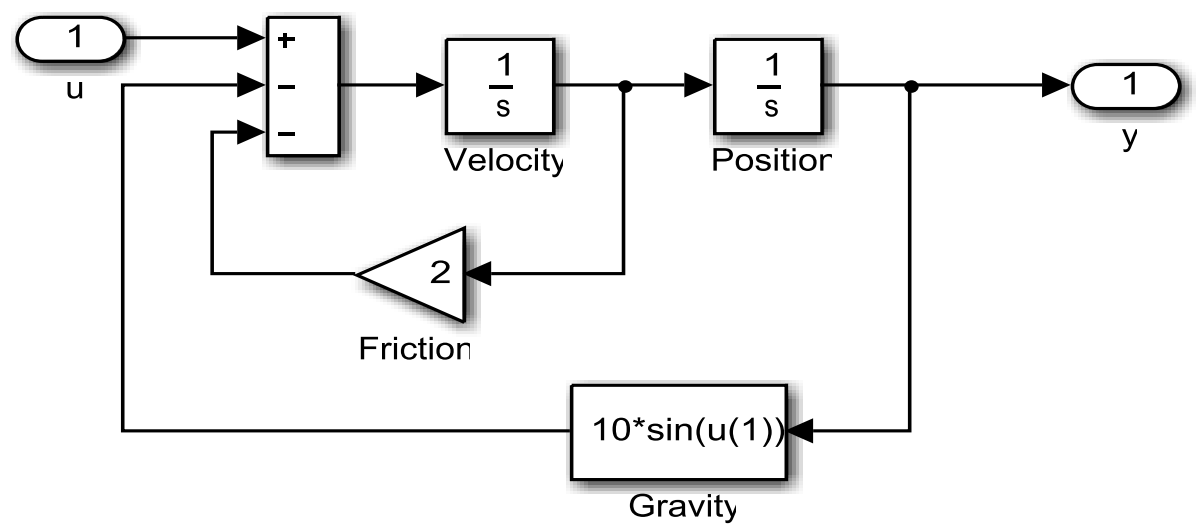

Fig 3.1: Simulink Model of the Robot Arm 


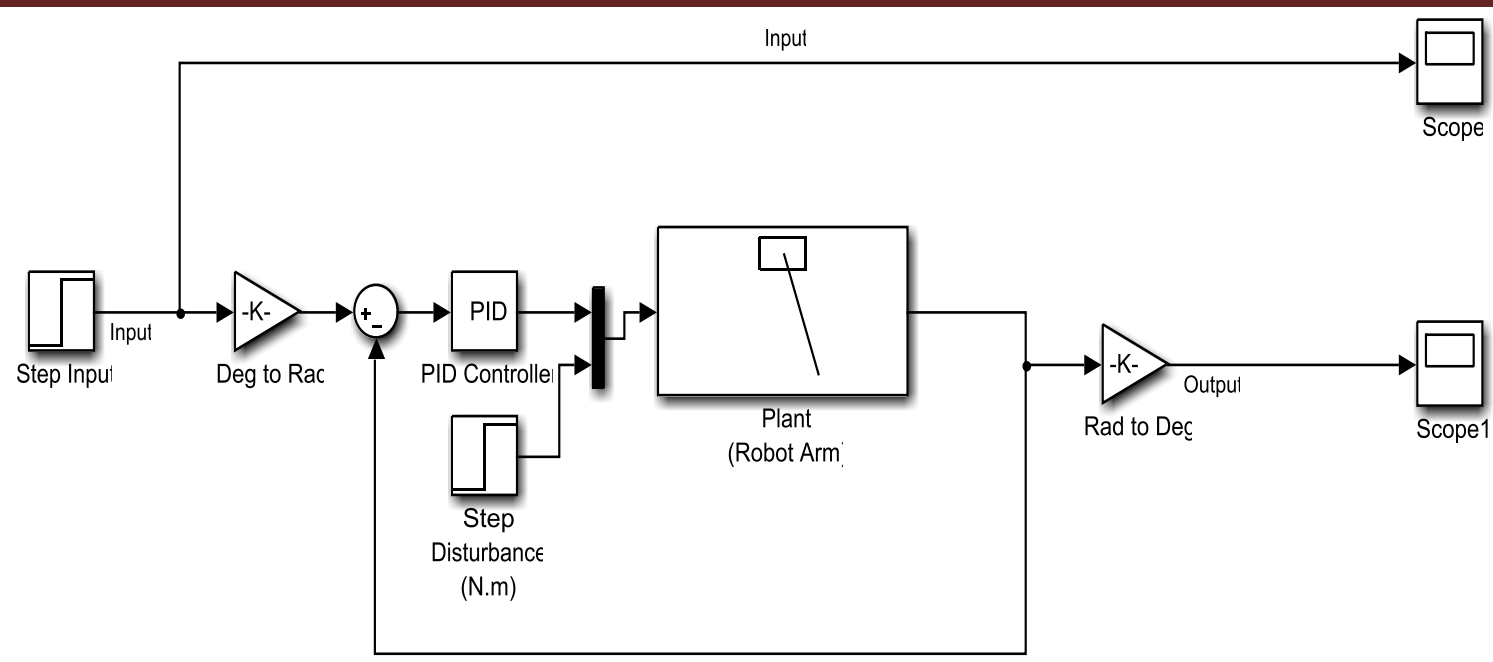

Figure 3.2: Simulink Model of Tunable PID controller

From Figure 3.2, it can be seen that external disturbance was introduced in the system using a mux block.

Since the PID controller is not adaptive and cannot be trained, simulation in the form of performance test was carried out to determine the ability of the PID controller in accommodating nonlinearities associated with the robot arm using step input. The performance of the system was determined in terms of time response and nonlinearities accommodation. The nonlinear parameter this work considered is voltage overshoot. Position profile as one of the controllable parameters was used. The reason for using position is that it is the parameter responsible for vibration cancellation in the system. Step input values in terms angular displacements (position) were used as input to the system. The input value of 60deg was chosen arbitrary.

\subsection{Result Presentation and Analysis}

Various simulations were carried out and the results obtained presented and analyzed. The gain was varied and system response is as shown in Figure 4.1. It could also be seen that from Figure 4.1, that there are still signs of variations in the signal and needs to be eradicated.

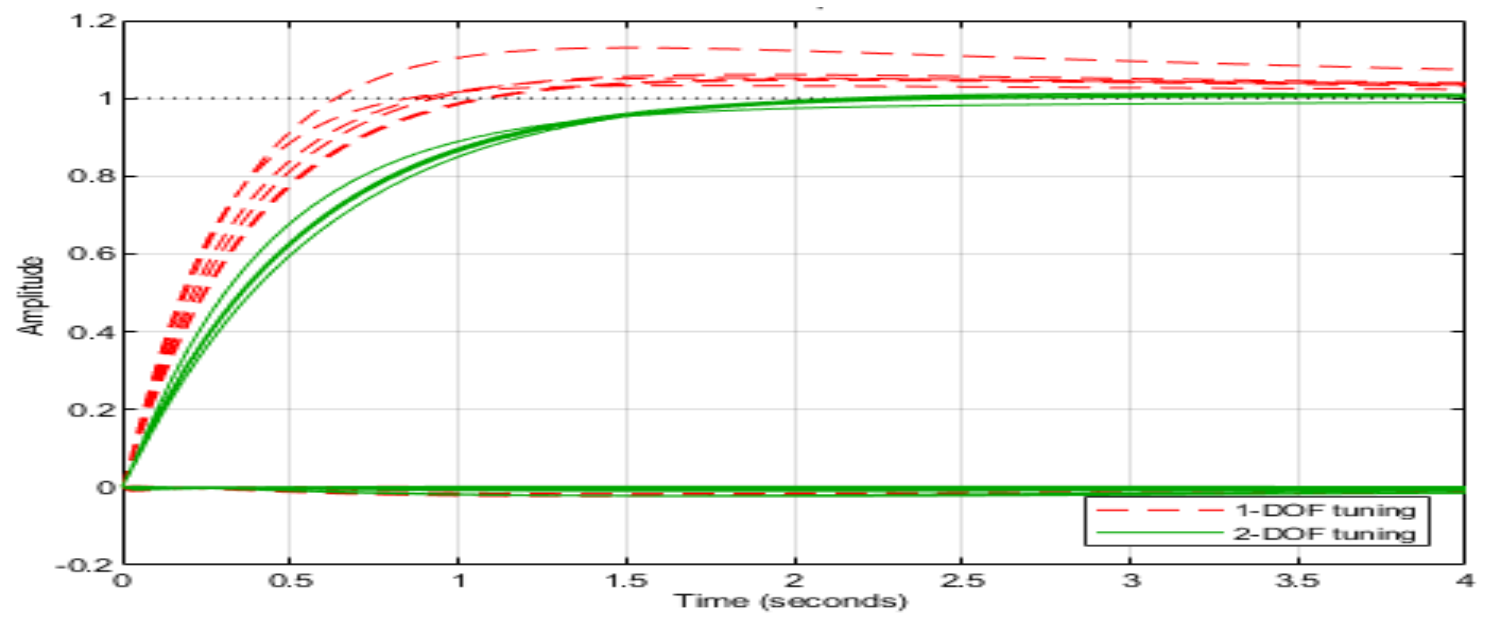

\section{1: Tuned response of the PID controller}


Those variations in signal are what translates to oscillations and finally to mechanical vibration. So, the PID system was further fine-tuned to produce the resultant effect as shown in figure 4.2 .

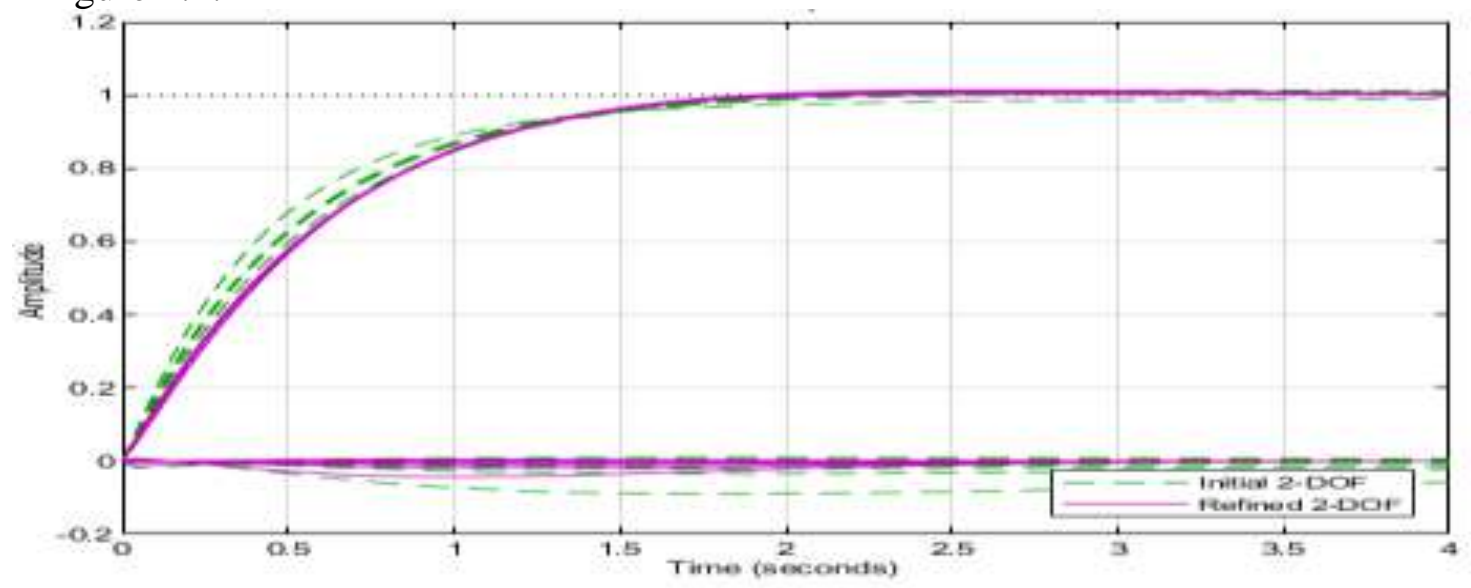

Figure 4.2: Fine tuning response of the manipulator

The set point tracking result for the tuned PID controller is presented in figure 4.9; showing the relative error (disturbance rejection analysis) at various amplitude signal, it can be seen that the tracking patterns (green) is not very accurate at certain times (sec). The accumulation of error brings inaccuracy in the speed of the manipulator thereby, affecting its motion. In this case, the system experienced overshot because the PID controller could not adapt to the target voltage of the system.

Final simulation result is as shown in Figure 4.3. From the figure, it can be seen that the target output of the PID did not closely follow the reference input. Result showed that the PID controller achieved steady state at $2.41 \mathrm{sec}$ and experienced voltage overshoot. From the graph, the percentage overshoot was calculated to be $38.46 \%$. These results imply that the PID system did not achieve a faster settling time, meaning that the system did not achieve steady state on time, which is not good for any real-time system or process. Also, during the period of oscillation as shown on the graph, the system experienced variation in the amplitude thereby introducing vibrations to the system.

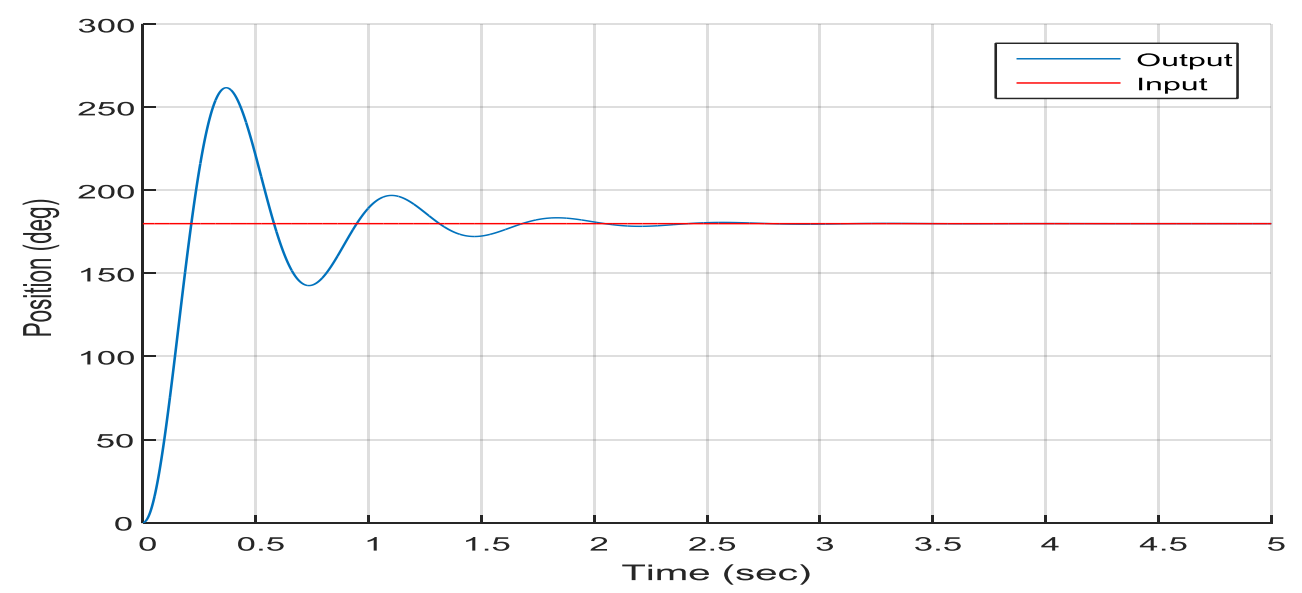

Figure 4.3: PID Controller Time Response

The set point tracking result for the tuned PID controller is presented in Figure 4.2; showing the relative error (disturbance rejection analysis) at various amplitude signal, it can be seen that the tracking patterns (green) is not very accurate at certain times $(\mathrm{sec})$. The accumulation of error brings inaccuracy in the speed of the manipulator thereby, affecting its motion. In 
this case, the system experienced overshot because the PID controller could not adapt to the target voltage of the system.

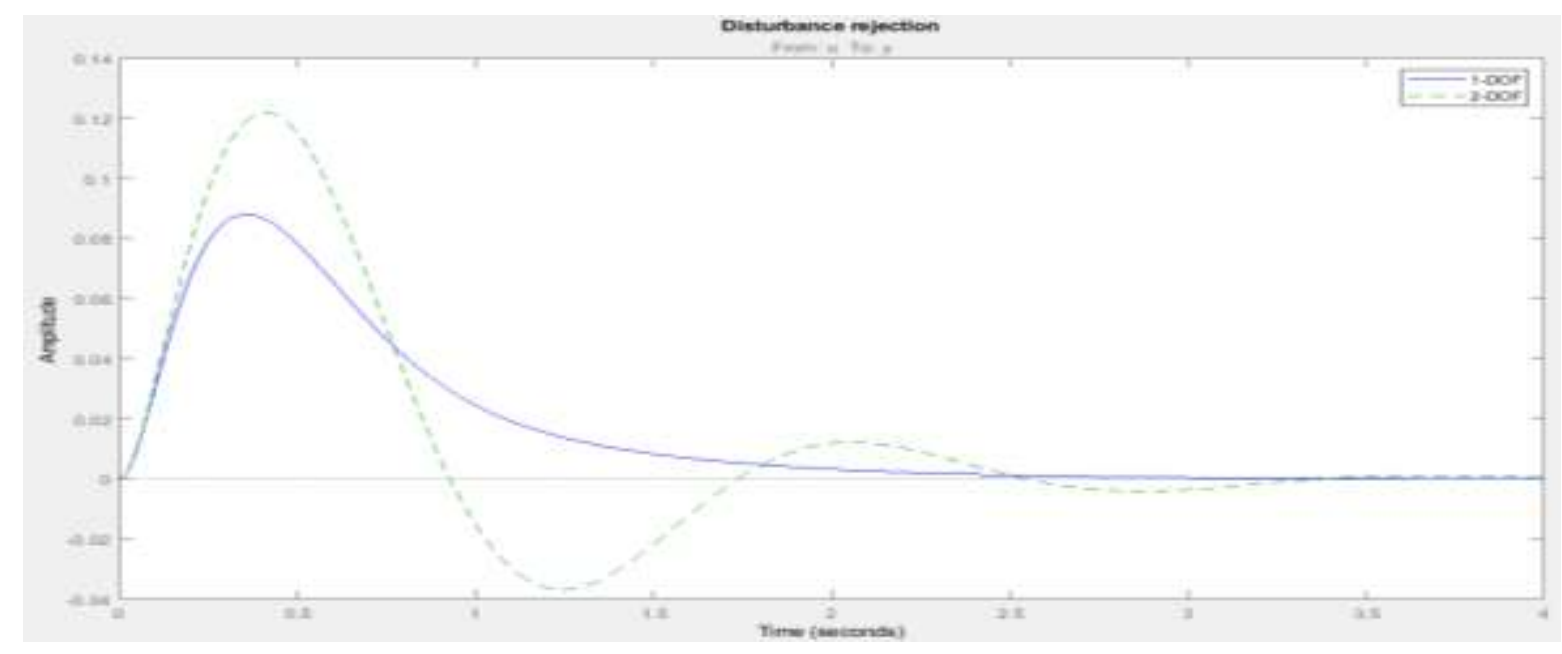

Figure 4.4: Tuned PID controller result

\subsection{Conclusion}

In this work, the design parameters required for the development of a tunable proportional integral derivative (TPID) controller for a robotic arm have been established. With the mathematical equations developed, the various steps taken were outlined and demonstrated. Even though PID has been good in the tuning of a robotic arm controller, it has a settling time which could not meet the need of a stable and responsive robot operation. Additionally, a high voltage overshoot shows poor stability and lack of required precision. The magnitude of the overshoot percentage implies a slow settling time. This is not particularly good for a realtime device in a system or process industry. The need for an intelligent-based and more reactive tuning method is to be the subject of future research in this dispensation.

\section{References}

1. P Arulmozhiand HaritaBaskar(2017), Development of Various Tunable PID Controllers for A Robotic Arm System, International Journal of Advanced Research in Basic Engineering Sciences and Technology (IJARBEST), Vol. 3, Special Issue 32, April 2017.

2. UmmeZakia, MehrdadMoallem and Carlo Menon (2019), PID-SMC Controller for A 2DOF Planar Robot, 2019 International Conference on Electrical, Computer and Communication Engineering (ECCE), 7-9 February, 2019.

3. M. Kamran Joyo, YarooqRaza, S. Faiz Ahmed, M. M. Billah, KushsairyKadir, Kanendra Naidu, Athar Ali and ZukhairiMohdYusof (2019), Optimized Proportional-IntegralDerivative Controller for Upper Limb Rehabilitation Robot, Electronics 2019, 8, 826; doi:10.3390/electronics8080826 www.m.

4. Astrom K. J. and Wittenmark B., (1989), “Adaptive Control”.Addison-Wesley.

5. Cheng, L., Hou, Z. G., Tan, M., Liu D., and Zou, A. M. (2008) Multi-agent based adaptive

6. consensus control for multiple manipulators with kinematic uncertainties, pp.189-194

7. D'Azzo, J.,Abdallah, C., Dawson, D., Dorato, P. and Jamshidi, M. (1991) Survey of robust control for rigid robots, IEEE Control Systems Magazines, pp.24-30

8. Farzin, P., Mina, M., Forouzan, S., Imam, N., and Sara, E. (2012) Design Baseline Computed 
9. Torque Controller, International Journal of Engineering (IJE), Volume (6): Issue (3), pp.129-130.

10. H. C. Inyiama and AgbarajiChukwudi Emmanuel (2015); Survey of Controller Design

11. Methods For A Robot Manipulator In Harsh Environments; NnamdiAzikiwe University Awka; NIGERIA

12. Kumar, R.G.U.V., and Raja, C.V.N. (2014) Comparison between FSC and PID Controller for

13. 5DOF Robot Arm, International Journal of Emerging Trends in Electrical and Electronics (IJETEE - ISSN: 2320-9569), Vol. 10, Issue. 2, pp.1-6

14. Ezzeldin M., Van Den Bosch P.P.J., and Waarsing R., (2009), "Improved convergence of MRAC design for printing system": IEEE Conference paper.

15. Jarzebowska., (2012), "Introduction to Nonlinear Control Theory": Modern Mechanics and Mathematics.

16. MathsWork.com., (2017), "Design Neural Network Predictive Controller in Simulink": Retrieved from https://www.mathworks.com/help/nnet/ug/design-neural-networkpredictive-controller-in-simulink.html.

17. Moussavi S. Z., Alasvandi M., Javadi S., and Morad E., (2014), "PMDC Motor Speed Control Optimization by Implementing ANFIS and MRAC": International Journal of Control Science and Engineering, Vol. 4 No. 1, pp. 1-8. doi: 10.5923.

18. Youns, M.D., Attya, S.M., and Abdulla, A.I. (2013) Position Control of Robot Arm Using Genetic Algorithm Based PID Controller, Al-Rafidain Engineering, Vol.21 No. 6, pp.1930 